Proceedings

\title{
Properties and Focusing Conditions of Laser Microplasma for Glass Structuring ${ }^{\dagger}$
}

\author{
Vladimir S. Rymkevich ${ }^{1, *}$, Anton I. Kiian ${ }^{2}$, Roman A. Zakoldaev ${ }^{3}$ and Maxim M. Sergeev ${ }^{4}$ \\ 1 ITMO University, St Petersburg 197101, Russia, kiya_anton@mail.ru (A.I.K.); zakoldaev@gmail.com \\ (R.A.Z.); maxim.m.sergeev@gmail.com (M.M.S.) \\ * Correspondence: v.rymkevich94@gmail.com \\ † Presented at 1st International Electronic Conference on Applied Sciences, 10-30 November 2020; Available \\ online: https://asec2020.sciforum.net/.
}

Published: 10 November 2020

\begin{abstract}
Currently, there is an increased interest among scientists in laser-induced methods (LIPAA, LIBDE, LIMP) for optical materials processing. The plume is formed due to the interaction of laser radiation transmitted through the glass sample with an absorbing target. In particular, laserinduced microplasma (LIMP) glass processing method development is realized by a plasma plume action arising at the interface of a carbon-based target and an optically transparent sample. Basically, the plasma plume can be considered as a spot tool that forms micro and nano-dimensional relief on the sample surface. This tool is needed to be investigated in the following way: geometry, divergence, and temperature. Detailed knowledge of these characteristics will improve the processing results. Here, we report on a detailed experimental study of glass processing by LIMP.
\end{abstract}

Keywords: laser microplasma; glass structuring; laser induced methods; LIMP; carbon

\section{Introduction}

Laser-induced methods based on the action of induced by laser radiation plasma plume are increasingly find various applications in a reason of the implementation simplicity, the plasma plume rigid attachment to the laser spot position, and high efficiency of energy transfer to the processed material [1,2]. An actual problem is to determine the relationship between the laser irradiation regime, the plasma plume properties, and the result of material processing. Since there is an "intermediary" between the laser radiation and the material, it transfers energy to the treatment zone, the solution of such a problem becomes very important. The problem is the precision of the fabrication of micro-optical and diffractive phase elements on a glass, as well as the increasing the resolution of the method [3].

This study considers the laser-induced microplasma (LIMP) application limited by a small laser spot, for processing fused silica glass, and also describes the effect of laser irradiation conditions on the spatial characteristics of the plasma plume and the relief geometry formed on the target and sample surfaces.

\section{Material and Methods}

In experiments to generate the plasma, we use graphite target with a thickness of at least $10 \mathrm{~mm}$ and the roughness $\mathrm{Rz}=5 \mu \mathrm{m}, \mathrm{Ra}=1.27 \mu \mathrm{m}$. The graphite surface is covered with fused silica glass $(1$ $\mathrm{mm}$ thickness and roughness $\mathrm{Rz}=180 \mathrm{~nm}, \mathrm{Ra}=25 \mathrm{~nm}$ ). The back side of glass is structured by the plasma plume, which is generated at the target-sample interface as a result of the action of laser radiation passing through the sample. As a laser source we apply nanosecond $\mathrm{Yb}$-fiber laser pulses with a duration of $50 \mathrm{~ns}$, a repetition rate of $40 \mathrm{kHz}$, and an energy per pulse after lens on target surface varying from $27 \mu \mathrm{J}$ to $136 \mu \mathrm{J}$. Processing is carried out by scanning at a constant movement 
speed $2 \mathrm{~mm} / \mathrm{s}$, which is fixed on the coordinate table. The laser spot at the interface between the two media is formed by lenses, the parameters of which are listed in Table 1. The longitudinal size of the waist $l_{f}$ is determined from the distance at which the waist radius increases by $5 \% .5 \mathrm{~mm}$-long tracks are fabricated on the sample surface with action of $10^{5}$ pulses.

Table 1. List of applied lenses for LIMP glass processing realization.

\begin{tabular}{cccc}
\hline Lens & $f_{\mathrm{wd}}, \mathbf{m m}$ & $\mathbf{l}_{\mathbf{f}} \mathbf{( 5 \% )}, \boldsymbol{\mu m}$ & $\mathbf{2 \omega _ { \mathbf { 0 } } , \boldsymbol { \mu m }}$ \\
\hline $4^{\times}, \mathrm{NA}=0.1$ & 31.40 & 15.68 & 13.9 \\
$10^{\times}, \mathrm{NA}=0.3$ & 17.13 & 4.77 & 7.7 \\
\hline
\end{tabular}

The formed structures in the form of tracks were examined using optical microscopy Carl Zeiss Axio Imager in differential integral contrast (DIC) mode of reflected light and with the contact profilometry on a HommelWerke T9000 with a depth probe accuracy of $10 \mathrm{~nm}$.

\section{Results}

As a result of laser plasma generation, a plasma plume is formed at the glass-target interface, (Figure 1a). Plasma plume consists of partially ionized carbon and oxygen atoms with a thermodynamic temperature $\sim 10,000 \mathrm{~K}$ [4]. In the absence of free space for expansion, the plasma begins to thermally act on the glass surface initiating the etching process in the affected area. By one pulse, a laser spot with a radius $\omega_{\mathrm{ob}}$ at the interface forms a cavity in a target with a radius $\mathrm{r}_{\mathrm{c}}$ and a depth $h_{c}$, and also generates a plasma plume, which etches in the sample a microlens with a radius $\mathrm{rg}_{\mathrm{g}}$ and deflection arrow $h_{\mathrm{g}}$ (Figure $1 \mathrm{~b}$ ). The microlens shifts the spot position. The result of this action is the track fabrication, which depth and transverse size are determined by the conditions of laser irradiation. The writing of a track with the same geometric characteristics along the entire length is achieved when the continuous plasma plume is formed, which does not decay between pulses. In this case, in addition to the glass etching, it is heated to the softening temperature, which will exclude the formation of cracks, and smothers the track edges. After processing by the LIMP method, a layer of graphite particles always remains on the sample, which must be removed before measurements. Removal of graphite particles can take place either by the heating in the furnace or by wet laser cleaning [5]. Cleaning in the oven is more time consuming, but avoids sample crackling due to an incorrectly selected mode in the wet laser cleaning method. In our case, we know a suitable wet laser cleaning mode for a given sample material.

In the experiment, we recorded several sets of lines, each of which differed in the position of the work plane. In the first case, the working plane was located at the interface between two media (T1), in the second, it was in the sample (T2), and the processing was carried out with diverging beams, in the third, the processing plane was in the target (T3), and the processing was in a converging beam. Micrographs and profilograms of segments recorded at $40 \mu \mathrm{J}$ pulse energy, but with different objectives, are presented in Figure 1c,d. 

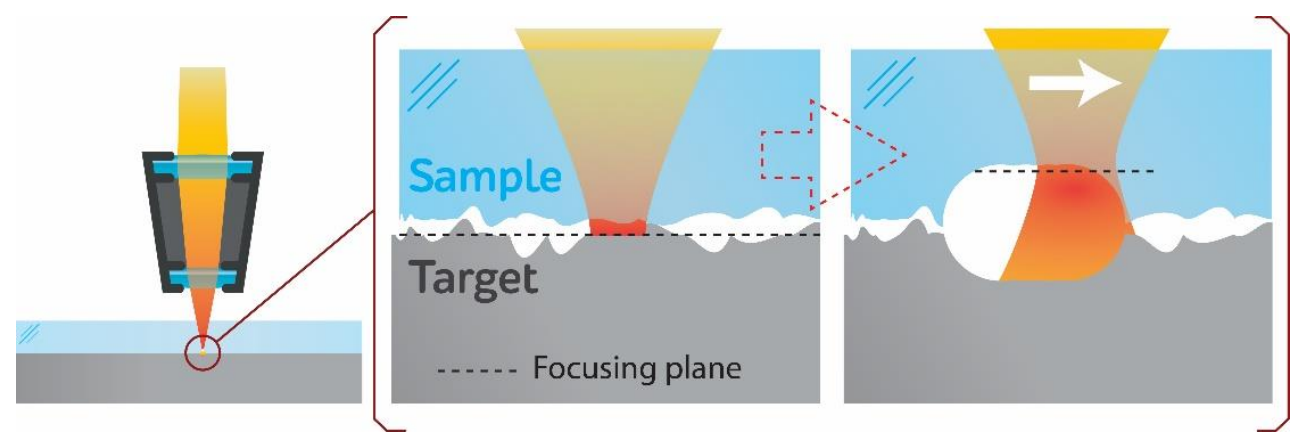

(a)

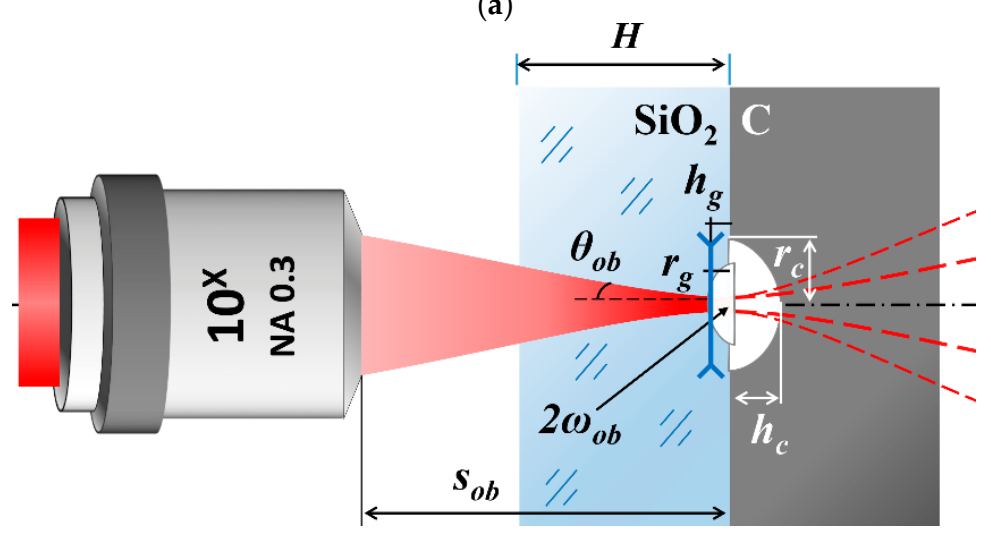

(b)
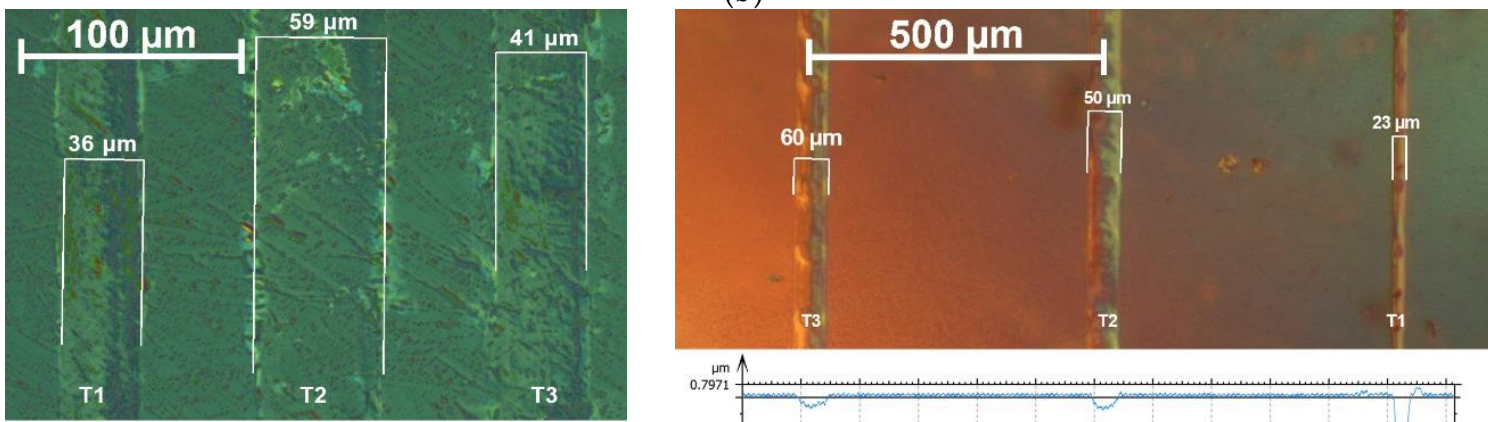

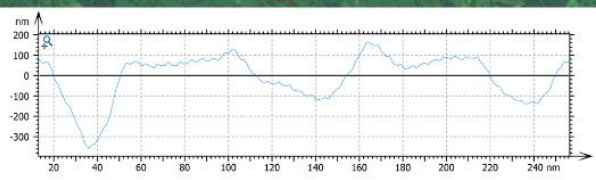

(c)

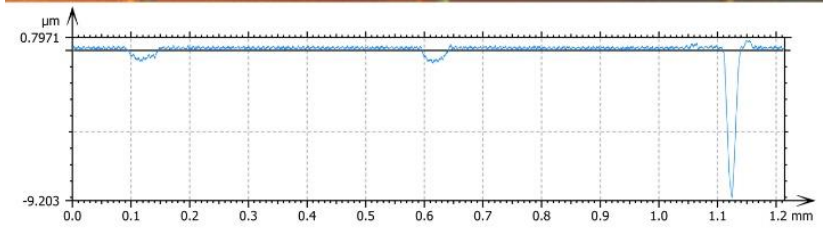

(d)

Figure 1. (a) Schematic representation of the processing area (b) Diagram of laser beam propagation from the objective to the processing plane (c) Optical micrograph in DIC mode and profilogram of recorded tracks on a $4^{\mathrm{x}}$ objective (d) Optical micrograph in DIC mode and profilogram of recorded tracks on a $10^{x}$ objective

\section{Discussion}

The micrographs (Figure 1c,d) show a strange behavior of tracks transverse dimensions recorded in the converging and diverging beams (T2 and T3). Between themselves, these two sets of tracks differ in the initial waist radius $\omega_{\mathrm{ob}}(13.9 \mu \mathrm{m}$ and $7.7 \mu \mathrm{m})$ and the divergence $\theta_{\mathrm{ob}}(73 \mathrm{mrad}$ and $133 \mathrm{mrad}$ ). The difference between the waist radius and the tracks dimensions could be explained by the plasma plume divergence. In each set, the displacement value of the focal plane in both directions (for T2 "-" and for T3 "+") relative to T1 was the same and equal to ZR of the lens used, i.e., the power density and spot size should be the same as the etched track sizeHowever, in a converging beam (T3), the track size was noticeably smaller than in a diverging beam (T2) (41 versus $59 \mu \mathrm{m}$ ). Considering that the initial T1 plane was set with taking into account the increase in the optical path in the sample, this phenomenon can only be explained by the influence of the microlens formed in the etch process. Indeed, depending on the microlens size and the $\mathrm{ZR}$ of the beam, both converging and diverging 
wavefront can incident on the microlens. Depending on the type of incident front, the microlens will either increase or decrease the spot size at the bottom of the ablating cavity in the target. This statement could be confirmed by the difference in the behavior of the same T2 and T3 tracks with a different radiation divergence (73 and $133 \mathrm{mrad}$ ). Similar dependences persisted when the pulse energy changed. One more conclusion can be drawn from the above: the closer the spot size is to the microlens size and the greater the radiation divergence, the greater the microlens effect on this radiation. The same microlens affects the energy density in the inducing radiation spot, and indirectly on the plasma plume divergence.

Let us calculate the energy required for etching the each entire track for these two sets. The total energy required is calculated based on the removed fused silica mass, determined by the volume, density and vaporization heat of this material $\left(\varrho=2200 \mathrm{~kg} / \mathrm{m}^{3}, \mathrm{LsiO2}=11770 \mathrm{~kJ} / \mathrm{kg}[6]\right)$. Table 2 shows data on tracks, their volume and the amount of energy required for evaporation.

Table 2. List of fused silica parameters and average decomposition energy per track.

\begin{tabular}{ccccc}
\hline Track & $\mathbf{S}, \boldsymbol{\mu m}^{\mathbf{2}}$ & $\mathbf{V}, \mathbf{1 0}^{\mathbf{4}} \cdot \boldsymbol{\mu m}^{\mathbf{3}}$ & Mass, $\mathbf{n g}$ & $\mathbf{E d e c}_{\text {de }} \mathbf{~ J}$ \\
\hline $4 \mathrm{x}, \mathrm{T} 1$ & 8.3 & 4.1 & 90.8 & 1.31 \\
$4 \mathrm{x}, \mathrm{T} 2$ & 9.5 & 4.7 & 103.9 & 1.50 \\
$4 \mathrm{x}, \mathrm{T} 3$ & 5.8 & 2.9 & 63.3 & 0.91 \\
\hline $10 \mathrm{x}, \mathrm{T} 1$ & 140.3 & 70 & 1543 & 22.25 \\
$10 \mathrm{x}, \mathrm{T} 2$ & 34.1 & 17 & 375.4 & 5.41 \\
$10 \mathrm{x}, \mathrm{T} 3$ & 29.9 & 15 & 328.8 & 4.74 \\
\hline
\end{tabular}

\section{Conclusions}

The paper considered the geometric characteristics dependence of the structures formed on the fused silica surface by working plane position of the laser radiation. It is shown that the resulting structure affects the laser radiation, which significantly changes the processing result. The effect nature depends on the parameters of the laser radiation itself, namely, the beam waist size and the divergence. Depending on the working plane displacement and the laser radiation divergence, both the narrowing effect and the expansion effect of the laser radiation, transmitted through the sample, can be achieved at the bottom of the crater in the target.

Author Contributions: Conceptualization, M.S.; Investigation V.R. and A.K.; Formal analysis, V.R.; Methodology, V.R.; M.S.; Writing-Original Draft, V.R.; Visualization, V.R.; Writing-Review \& Editing, M.S., R.Z.; Supervision, M.S.; Project administration, M.S.; Funding acquisition, R.Z. All authors have read and agreed to the published version of the manuscript.

Funding: The study is funded by the grant of Russian Science Foundation (project № 20-71-10103)

Conflicts of Interest: The authors declare no conflict of interest.

\section{References}

1. Xu, S.; Bin, L.; Pan, C.; Ren, L.; Tang, B.; Hu, Q.; Jiang, L. Ultrafast Fabrication of Micro-Channels and Graphite Patterns on Glass by Nanosecond Laser-Induced Plasma-Assisted Ablation (Lipaa) for Electrofluidic Devices. J. Mater. Proc. Technol. 2017, 247, 204-213.

2. Xie, X.Z.; Li, M.N.; Long, Y.J.; Li, J.G.; Zhou, C.X.; Zou, Z.X. Laser-Induced Backside Wet/Dry Etching Microstructures on Transparent and Brittle Materials. In Proceedings of the Advanced Laser Processing and Manufacturing III 2019, Hangzhou, China, 21-22 October 2019.

3. Shkuratova, V.; Vladimir R.; Galina, K.; Maksim, S. Laser-Induced Microplasma as Effective Tool for Phase Elements Fabrication on Amorphous and Crystalline Materials. J. Laser Micro Nanoeng. 2018, 13, $211-215$.

4. Veiko, V.P.; Sergei, A.V.; Roman, A.Z.; Maksim, M.S.; Andrei, A.S.; Galina, K.K.; Konstantin, A.M. LaserInduced Microplasma as a Tool for Microstructuring Transparent Media. Quantum Electronics 2017, 47, 842.

5. Sergeev, M.M.; Zakoldaev, R.A.; Kostyuk, G.K. Changes in the Spectral Characteristics of Quartz-Glass Plates When They Are Processed with Laser-Induced Plasma. J. Opt. Technol. 2017, 84, 447-452. 
6. Kraus, R.G.; Sarah, T.S.; Swift, D.C.; Bolme, C.A.; Smith, R.F.; Hamel, S.; Hammel, B.D.; Spaulding, D.K.; Hicks, D.G.; Eggert, J.H. Shock Vaporization of Silica and the Thermodynamics of Planetary Impact Events. J. Geophys. Res. Planets 2012, 117, E9.

Publisher's Note: MDPI stays neutral with regard to jurisdictional claims in published maps and institutional affiliations.

(c) 2020 by the authors. Submitted for possible open access publication under the terms and conditions of the Creative Commons Attribution (CC BY) license (http://creativecommons.org/licenses/by/4.0/). 